\title{
The Hubble Tension: Change in Dark Energy or a Case for Modified Gravity?
}

\author{
C Sivaram \\ Indian Institute of Astrophysics, Bangalore - 560 034, India \\ e-mail: sivaram@iiap.res.in
}

\section{Kenath Arun ${ }^{1}$}

Christ Junior College, Bangalore - 560 029, India e-mail: kenath.arun@cjc.christcollege.edu

Orcid ID: 0000-0002-2183-9425

\section{Louise Rebecca}

Christ Junior College, Bangalore - 560 029, India e-mail: louise.rheanna@cjc.christcollege.edu

\begin{abstract}
Here we propose a modification of the gravitational field on large scales as an alternate explanation for the discrepancy in the value of the Hubble constant as implied by Planck observations of the CMBR in the early Universe and that deduced from other distance indicators in the present epoch.
\end{abstract}

Keywords: Hubble tension; modified gravity; dark energy; dark matter

\footnotetext{
${ }^{1}$ Corresponding author
} 


\section{Introduction}

Recently much controversy has been raised about the cosmological conundrum involving the discrepancy in the value of the Hubble constant as implied by Planck satellite observations of the CMBR in the early Universe and that deduced from other distance indicators (standard candles like SN, tip of RG branch, etc.) in the present epoch. The Planck estimate is about $67 \mathrm{~km} / \mathrm{s} / M p c$ [1], while that deduced from distance indicators at the present epoch is around $73-74 \mathrm{~km} / \mathrm{s} / \mathrm{Mpc}$ [2], and the independent determination of the local value of the Hubble constant based on a calibration of the Tip of the Red Giant Branch (TRGB) applied to Type Ia supernovae found a value of $69.8 \mathrm{~km} / \mathrm{s} / \mathrm{Mpc}$ [3]. The Hubble constant measures the rate of expansion of the Universe so that the above values suggest that the Universe is expanding faster at present than at the time of the CMBR epoch.

It is implied by Reiss and others [4] that this is a real discrepancy and not due to observational uncertainties. The difference amounts to about ten per cent in the value of the expansion rate, well above the errors and uncertainties. Several suggestions have been made as to the cause of such a discrepancy. Perhaps a new kind of matter or unknown particle has now become more dominant, adding to the 'push' in the expansion rate. For instance, a recent suggestion [5] involves a calculation of the amount of change in the quantum fields, needed to account for the dark energy (DE) change. This quantum field causing a change in the DE implies the existence of a new particle with a mass roughly that of the axion (about $10^{-3} \mathrm{eV}$ ), already predicted earlier. As the background density decreases this particle is now more dominant.

\section{Change in Hubble constant}

In general we can write for a change in Hubble constant $(d H)$ relating it to a change in density $d \rho$ as:

$2 H d H=\frac{8 \pi G}{3} d \rho$

Here, $H=70 \mathrm{~km} / \mathrm{s} / M p c, d H=5 \mathrm{~km} / \mathrm{s} / M p c$. This gives, $2 H d H=10^{-36} \mathrm{~cm}^{2} / \mathrm{s}^{2} / \mathrm{cm}^{2}$, which from equation (1) gives:

$d \rho \approx 2 \times 10^{-30} \mathrm{~g} / \mathrm{cc}$

This is the change in density that gives the change in Hubble constant. The quantum field energy density is given as $\frac{m}{(\hbar / m c)^{3}}$, i.e. a pair of particles separated by Compton length, where the mass causing the change in the quantum field energy density is $m$. Hence we have: 
$d \rho=\frac{m^{4} c^{3}}{\hbar^{3}}$

Equations (1) - (3) imply:

$m \approx 10^{-36} \mathrm{~g}=10^{-4} \mathrm{eV}$

Equation (1) also implies that a change in $H$ could be due to a change in the gravitational constant, rather than a change in density. This would require an increase in $G$ by about 10 per cent over a few billion years. This would contradict solar system measurements, i.e. from radar ranging of interplanetary spacecraft putting limits on varying $G$ theories [6]. However if not connected with dark energy, change in $G$ can still affect $H$ as in equation (1), if there is just matter density dominating at higher $z$. This would however require increase in $G$ rather than decrease as in the usual theory. A decrease of $G$ with epoch could increase the DE density since:

$\rho_{\Lambda}=\frac{\Lambda c^{2}}{8 \pi G}$

However as on the RHS of equation (1) we have $G$ multiplying $\rho$, this would cancel out the $G$ variation, i.e. $G \rho_{\Lambda}=$ constant.

\section{Resolution of Hubble tension through modified gravity}

We point out that a form of modified gravity - used in recent work [7-9] as a way to account for flat rotation curves of galaxies without invoking DM - could in principle account also for the increase in $H$. This also accounts for the DM needed in galaxy clusters $[8,9]$ and is consistent with other observations of galaxies in the early Universe (which are more consistent with MOND [10]). In this work we invoked a minimal acceleration in the gravitational field, $a_{0}$, which implies a modification of gravity over a scale larger than $R_{C}$, $\left(R_{C}=\left(\frac{G M}{a_{0}}\right)^{1 / 2}\right), M$ the mass of the system - galaxy or cluster. Again the gravitational selfenergy density given by $\kappa(\nabla \phi)^{2}$ becomes important if $\rho$ drops below certain value. So that the modified Poisson equation is:

$\nabla^{2} \phi+\kappa(\nabla \phi)^{2}=4 \pi G \rho$

For the self-energy term to become important the equation $\nabla^{2} \phi+\kappa(\nabla \phi)^{2}=0$, has the solution [11]:

$\phi=\frac{G M}{r}+\kappa^{\prime} \ln \frac{R}{R_{C}}$

The extra term accounts for flat rotation curves and also for outer rotation velocities of super spiral galaxies. The extra term in the Poisson equation (equation (6)) and its solution 
given by equation (7) could have interesting consequences for current observations of super spirals [12], wherein their large extent (450,000 light-years) is associated with large rotation velocities of up to $\sim 450 \mathrm{~km} / \mathrm{s}$ at their periphery. Conventionally such large velocity would imply a large amount of DM, i.e. about $\sim 10^{13} M_{\odot}$. However, the extra term in our model would give a velocity given by:

$v=\left(G M a_{0}\right)^{1 / 4}\left(\ln \frac{R}{R_{C}}\right)^{1 / 2}$

where $R_{C}$ corresponds to the radius at which acceleration approaches $a_{0}$. With $R_{C}=20 \mathrm{kpc}$ and super spiral extant $R_{S S} \approx 200 \mathrm{kpc}$, this would give velocities $\sim 450 \mathrm{~km} / \mathrm{s}$. I.e., the logarithmic term makes gravity stronger above $R_{C}$ (i.e., potential going as $\ln R$, instead of $1 / R$ ), so that we do not need such colossal amounts of DM.

The usual Friedmann equation now gets modified to:

$\frac{\dot{R}^{2}}{R^{2}}=\frac{8 \pi G \rho}{3}+\left(G M a_{0}\right)^{1 / 2} \ln \frac{R}{R_{C}}$

where $R_{C}$ is the scale factor at which modification becomes relevant.

The second term can be seen as a modification in potential energy due to gravitational self-energy density in the usual balance between kinetic energy term and potential energy term, $\frac{8 \pi G \rho}{3}$ (in the expanding Universe), i.e. the usual Newtonian analogue agreeing with the GR result.

With $a_{0}=10^{-8} \mathrm{~cm} / \mathrm{s}^{2}$, and the Universe having expanded at present to $R>R_{\min }$ (where $R_{\min } \approx 10^{28} \mathrm{~cm}$ ) the modified term will also contribute. Putting values of $R \approx 2 \times$ $10^{28} \mathrm{~cm}, \rho \approx 10^{-29} \mathrm{~g} / \mathrm{cc}$, and the mass of the Universe, $M=2 \pi^{2} R^{3} \rho \approx 10^{56} \mathrm{~g}$, the usual first term is $\approx 10^{21}$, whereas the second term $\approx 10^{20}$. This would suggest that this extra term now manifesting itself would cause an increase of the expansion rate, i.e. a change in Hubble constant by about five per cent. This could perhaps account for the faster expansion rate seen at the present epoch.

\section{Conclusion}

We suggest that this particular modification of the gravitational field on large scales (low accelerations), used earlier to account for rotation curves of galaxies and for cluster dynamics, could well provide an alternate explanation for the discrepancy and could perhaps help in relaxing the Hubble tension. We also predict that as this extra term, increases slowly with the logarithm of the scale, it would imply an even higher value of $H$ at later epochs. 


\section{References:}

[1] Aghanim, N. et al.: Planck 2018 results. VI. Cosmological parameters. arXiv:1807.06209v2 [astro-ph.CO] (2018)

[2] Riess, A. et al.: Large Magellanic Cloud Cepheid standards provide a 1\% foundation for the determination of the Hubble constant and stronger evidence for physics beyond $\Lambda \mathrm{CDM}$. Astrophys. J. 876, 85 (2019)

[3] Freedman, W. L. et al.: The Carnegie-Chicago Hubble Program. VIII. An Independent Determination of the Hubble Constant Based on the Tip of the Red Giant Branch. Astrophys. J. 882, 34 (2019)

[4] D'Arcy Kenworthy, W., Scolnic, D. and Riess, A.: The local perspective on the Hubble tension: local structure does not impact measurement of the Hubble constant. Astrophys. J. 875,145 (2019)

[5] Cerdonio, M.: The H0 tension: did a QCD meV axion emerge? arXiv:1906.07080v3 [grqc] (2019)

[6] Genova, A. et al.: Solar system expansion and strong equivalence principle as seen by the NASA MESSENGER mission. Nature Commun. 9, 289 (2018)

[7] Sivaram, C.: Dark matter (energy) may be indistinguishable from modified gravity (MOND). Int. J. Mod. Phys. D 26, 1743010 (2017)

[8] Rebecca, L., Arun, K. and Sivaram, C.: Dark energy constraints on masses and sizes of large scale cosmic structures. Astrophys. Space Sci. 363, 149 (2018)

[9] Rebecca, L., Arun, K. and Sivaram, C.: Dark matter density distributions and dark energy constraints on structure formation including MOND. Indian J. Phys. (2019). https://doi.org/10.1007/s12648-019-01591-8

[10] Milgrom, M.: High-redshift rotation curves and MOND. arXiv:1703.06110v3 [astroph.GA] (2017)

[11] Sivaram, C., Arun, K. and Rebecca, L.: MOND, MONG, MORG as alternatives to dark matter and dark energy, and consequences for cosmic structures. J. Astrophys. Astron. 41, 4 (2020)

[12] Ogle, P. M. et al.: A break in spiral galaxy scaling relations at the upper limit of galaxy mass. Astrophys. J. Lett. 884, L11 (2019) 